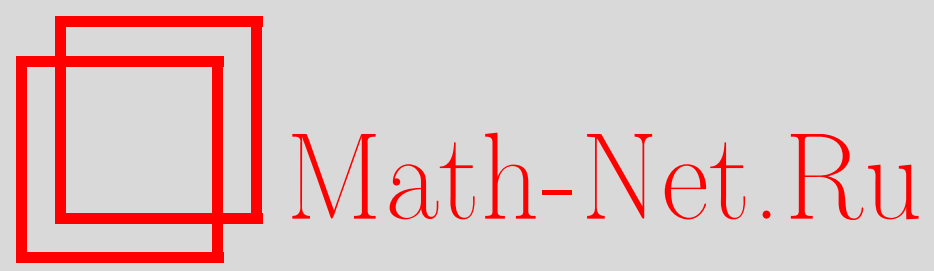

В. О. Бытев, И. В. Слезко, Исследование напряженно-деформированного состояния асимметрично-упругих пластин, Вестн. Сам. гос. техн. ун-та. Сер. Физ.-мат. науки, 2011, выпуск 1(), 186-190

DOI: https://doi.org/10.14498/vsgtu886

Использование Общероссийского математического портала Math-Net.Ru подразумевает, что вы прочитали и согласны с пользовательским соглашением http: //www. mathnet.ru/rus/agreement

Параметры загрузки:

IP : 54.174 .149 .18

26 апреля 2023 г., $17: 18: 58$ 


\title{
УДК 539.371
}

\section{ИССЛЕДОВАНИЕ НАПРЯЖЁННО-ДЕФОРМИРОВАННОГО СОСТОЯНИЯ АСИММЕТРИЧНО-УПРУГИХ ПЛАСТИН}

\author{
В.О. Бытев, И. В. Слезко
}

Тюменский государственный университет, 625003, Тюмень, ул. Семакова, 10.

E-mail: slezkoirina@rambler.ru

Рассмотрена двумерная линейная модель дебормаиии асимметрично-упругого тела. Приведено точное решение задачи плоской асимметричной теории упругости о чистом сдвиге в пластине, ослабленной отверстием. Проведён сравнительный анализ полученного решения с классическим.

Ключевые слова: асимметричная упругость, деформащия пластин, чистый сдвиг.

Введение. Методы теории упругости и её приложений применяются в различных областях науки и техники. Задачи, которые удаётся решать в рамках линейной теории, разнообразны. Однако, как известно, линейная теория не даёт возможности вести расчёт изделий из полимерных материалов. В связи с этим актуальной задачей является разработка такой модели упругой деформации, которая позволила бы применять наиболее простые математические методы и приёмы при решении задач об исследовании напряжённо-деформированного состояния тела, изготовленного из эластичного материала.

1. Модель асимметричной упругости. Как известно [1], уравнения статики имеют вид

$$
\operatorname{div} T+\boldsymbol{F}=0,
$$

где $T$ - тензор напряжений, $\boldsymbol{F}$ - вектор объёмных сил. Общий вид тензора напряжений установлен в результате решения задачи групповой классификации законов сохранения в работах $[2,3]$. В случае плоской задачи он имеет вид:

$$
T=I \lambda_{0} \operatorname{div} \boldsymbol{u}+M D_{0},
$$

где $I-$ единичный тензор, $\boldsymbol{u}=\{u, v\}$ - вектор смещений, $D_{0}$ - девиатор деформаций,

$$
M=\left(\begin{array}{cc}
\mu & \mu_{0} \\
-\mu_{0} & \mu
\end{array}\right)
$$

$\lambda_{0}, \mu, \mu_{0}$ - кинетические коэффициенты, причём $\lambda_{0}>0, \mu>0$, а $\mu_{0} \in \mathbb{R}$.

Данная модель служит обобщением классической линейной модели деформации упругого тела. Так, если положить в (1), (2) $\mu_{0}=0, \lambda_{0}=\lambda+\mu$, считая при этом $\lambda$ и $\mu$ параметрами Ламе, то получим известную зависимость между тензорами напряжений и деформаций [1].

Поясним суть появления дополнительного третьего параметра в модели. Обозначим через $\vec{\tau}^{0}=\left(M D_{0} \cdot \vec{n}\right)$ - вектор девиатора тензора $T$, а через

Владислав Олегович Бытев (д.ф.-м.н., проф.), зав. кафедрой, каф. математического моделирования. Ирина Викторовна Слезко (к.ф.-м.н.), доцент, каф. математического моделирования. 
$\vec{d}^{0}=\left(D_{0} \cdot \vec{n}\right)$ - вектор тензора $D_{0}$. Согласно [3]

$$
\cos \gamma=\frac{\left(\vec{\tau}^{0} \cdot \vec{d}^{0}\right)}{\left|\vec{\tau}^{0}\right| \cdot\left|\vec{d}^{0}\right|}=\frac{\mu}{\sqrt{\mu^{2}+\mu_{0}^{2}}}=\frac{1}{\sqrt{1+\left(\mu_{0} / \mu\right)^{2}}} .
$$

Последнее соотношение определяет меру несоосности тензоров $M D_{0}$ и $D_{0}$.

Из формул (1), (2) выпишем выражения компонент симметричного тензора напряжений $\sigma_{i j}$ через компоненты симметричного тензора деформаций $\varepsilon^{i j}$ :

$$
\begin{aligned}
& \sigma_{11}=\left(\lambda_{0}+\mu\right) \varepsilon^{11}+2 \mu_{0} \varepsilon^{12}+\left(\lambda_{0}-\mu\right) \varepsilon^{22}, \\
& \sigma_{12}=-\mu_{0} \varepsilon^{11}+2 \mu \varepsilon^{12}+\mu_{0} \varepsilon^{22}, \\
& \sigma_{22}=\left(\lambda_{0}-\mu\right) \varepsilon^{11}-2 \mu_{0} \varepsilon^{12}+\left(\lambda_{0}+\mu\right) \varepsilon^{22} .
\end{aligned}
$$

Здесь

$$
\varepsilon^{i j}=\frac{1}{2}\left(\frac{\partial u_{i}}{\partial x_{j}}+\frac{\partial u_{j}}{\partial x_{i}}\right) .
$$

Рассмотрим матрицу коэффициентов системы (3):

$$
A=\left(\begin{array}{ccc}
\lambda_{0}+\mu & 2 \mu_{0} & \lambda_{0}-\mu \\
-\mu_{0} & 2 \mu & \mu_{0} \\
\lambda_{0}-\mu & -2 \mu_{0} & \lambda_{0}+\mu
\end{array}\right)
$$

Итак, в линейной модели асимметричной упругости симметричные тензоры напряжений и деформаций связаны несимметричным преобразующим тензором с матрицей $A$-это принципиальное отличие данной модели от классической модели линейной теории упругости.

Следует отметить, что модель асимметричной упругости не связана с несимметричной моментной упругостью. Для последней характерно введение моментных напряжений, несимметричность тензоров напряжений и деформаций, наличие большого числа кинетических коэффициентов и др.

Вычислим $\operatorname{det} A=2 \lambda_{0} \kappa_{0}^{2} \neq 0, \kappa_{0}^{2}=\mu^{2}+\mu_{0}^{2}$. Матрица $A$ - невырожденная, следовательно, найдутся обратные зависимости компонент тензора деформаций от компонент тензора напряжений.

Как и в классическом случае [1], можно выразить вектор смещений и компоненты тензора напряжений через комплексные потенциалы $\varphi(z), \psi(z)$, вводя функцию Эри $U(x, y)$ по формулам

$$
\sigma_{11}=U, y y, \quad \sigma_{12}=-U, x y, \quad \sigma_{22}=U, x x,
$$

где запятая означает дифференцирование по соответствующим переменным.

Комплексное представление вектора смещений имеет вид

$$
2 \kappa_{0}^{2}(u+i v)=\left(2 \lambda_{0}^{-1} \kappa_{0}^{2}+\bar{\kappa}\right) \varphi(z)-\kappa z \overline{\varphi^{\prime}(z)}-\kappa \overline{\psi(z)},
$$

где $\kappa=\mu+i \mu_{0}$.

Рассмотрим теперь две комплексные плоскости $Z, \Omega$ и зададим конформное отображение $z=\omega(\zeta)$, которое переводит область $\mathrm{S} \subset \mathrm{Z}$ в область $\Sigma \subset \Omega$. 
Введём на плоскости $\Omega$ полярные координаты $(\rho, \vartheta)$ так, что $\omega=\rho e^{i \vartheta}$. Приведём выражения компонент вектора смещений с помощью комплексных потенциалов $\varphi(\zeta)$ и $\psi(\zeta)$ в полярных координатах:

$$
2 \kappa_{0}^{2}\left(u_{\rho}+i u_{\vartheta}\right)=e^{-i \vartheta}\left[\left(2 \lambda_{0}^{-1} \kappa_{0}^{2}+\bar{\kappa}\right) \varphi(\zeta)-\kappa \zeta \overline{\varphi^{\prime}(\zeta)}-\kappa \overline{\psi(\zeta)}\right] .
$$

Как уже было отмечено, асимметричная модель является обобщением классических формул Лява. При $\mu_{0}=0$ и $\lambda_{0}=\lambda+\mu$ имеем

$$
2 \mu\left(u_{\rho}+i u_{\vartheta}\right)=e^{-i \vartheta}\left[\kappa \varphi(\zeta)-z \overline{\varphi^{\prime}(\zeta)}-\overline{\psi(\zeta)}\right],
$$

где $\kappa=(\lambda+3 \mu) /(\lambda+\mu)=3-4 \sigma ; \lambda, \mu$ - модули упругости $(\lambda>0, \mu>0) ; \sigma-$ коэффициент Пуассона.

Поле напряжений обеих моделей одинаково. Уравнения на компоненты тензора напряжений в комплексной формулировке имеют следующий вид:

$$
\sigma_{11}+\sigma_{22}=4 \operatorname{Re}\left[\varphi^{\prime}(z)\right], \quad \sigma_{22}-\sigma_{11}+2 i \sigma_{12}=2\left[\bar{z} \varphi^{\prime \prime}(\zeta)+\psi^{\prime}(\zeta)\right] .
$$

Сделаем ещё одно замечание, касающееся обозначений. Для кинетических параметров асимметричной модели вводятся обозначения $\lambda_{0}, \mu, \mu_{0}, \kappa$. Эти параметры никак не связаны с параметрами Ламе и известна только область их изменения. Чтобы получить возможность сравнивать модель асимметричной упругости с классической линейной моделью, полагаем $\lambda_{0}=\lambda+\mu$, и кинетические коэффициенты принимают привычный смысл модулей упругости.

Использование асимметричной модели даёт возможность вести расчёт изделий из полимеров, получая при этом адекватные результаты.

2. Деформация асимметрично-упругих пластин. В качестве примера плоской деформации упругих пластин в рамках классической и асимметричной моделей рассмотрим задачу о чистом сдвиге в пластине, ослабленной отверстием в форме квадрата с закруглёнными углами.

Будем пользоваться конформным отображением внешности отверстия на внутренность круга единичного радиуса. Возьмём отображающую функцию, удерживая в ней два члена [4]:

$$
z=\omega(\zeta)=R\left(1 / \zeta-\zeta^{3} / 6\right)
$$

Здесь $R=3 a / 5$, где $a$ - сторона квадрата.

Согласно [4], выражения для комплексных потенциалов задаются следующими уравнениями:

$$
\varphi(\zeta)=i \frac{6}{5} R \tau, \quad \psi(\zeta)=-i R \tau\left[\frac{1}{\zeta}+\frac{13 \zeta^{3}}{5\left(2+\zeta^{4}\right)}\right]
$$

где $\tau$-сдвиговое усилие. Для данной задачи получено поле напряжений и выражения для компонент вектора смещений модели (4) и, соответственно, (5). Их выражения громоздки, поэтому здесь они не приводятся.

Проиллюстрируем полученное решение на примере задачи о чистом сдвиге пластины из резины. Физические характеристики материала следующие: 
коэффициент Пуассона $\sigma=0,46$, модуль упругости $E=1,5 \cdot 10^{6} \mathrm{H} / \mathrm{m}^{2}$. Сторона квадрата $a=0,6 \cdot 10^{-3} \mathrm{M}$, ширина пластины $-25 \cdot 10^{-3} \mathrm{M}$, сдвиговое усилие $\tau=0,025 \mathrm{kH}$.

На рис. 1 представлена форма деформированного отверстия, полученная с использованием классической линейной модели. Видим подтверждение того, что классическая теория не позволяет получать адекватные результаты при её применении к полимерным материалам (отверстие принимает неестественную форму - образуются углы, петли).

При использовании асимметричной модели при $\mu_{0}=1 \cdot 10^{6} \mathrm{H} / \mathrm{m}^{2}$ получаем иную деформацию отверстия (см. рис. 2): в результате действия нагрузки деформация отверстия происходит без образования углов и петель. Отметим, что при увеличении нагрузки, а в реальном эксперименте это может соответствовать уменьшению модуля упругости материала, асимметричная модель продолжает давать физически приемлемые результаты. То есть при введении дополнительного параметра в модель она может быть использована и для более эластичных материалов.

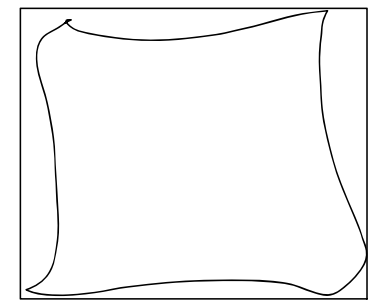

Рис. 1. Деформация отверстия (классическая модель)

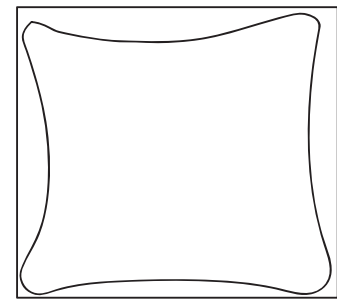

Рис. 2. Деформация отверстия (асимметричная модель, $\tau=0,025 \mathrm{kH})$

Геометрическая интерпретация полученного решения позволяет говорить о том, что использование асимметричной модели для описания деформированного состояния тела даёт возможность получать физически реальные результаты в рамках линейной теории упругости для эластичных материалов. Очевидно, реальные значения дополнительного модуля упругости могут быть получены экспериментально. Вычислительный эксперимент показывает, что величина параметра $\mu_{0}$ сравнима со значением модуля Юнга.

Итак, получен в распоряжение дополнительный модуль упругости, который позволяет расширить класс задач, решаемых в рамках линейной теории.

\section{БИБЛИОГРАФИЧЕСКИЙ СПИСОК}

1. Мусхелишвили Н. И. Некоторые основные задачи математической теории упругости. М.: Наука, 1966. 707 с.; англ. пер.: Muskhelishvili N. I. Some basic problems of the mathematical theory of elasticity. Basic equations, the plane theory of elasticity, torsion and bending. Leyden: Noordhoff International Publishing, 1977. 768 pp.

2. Аннин Б.Д., Бытев В.О., Сенашев С.И. Групповые свойства уравнений упругости и пластичности. Новосибирск: Наука. Сибир. отд., 1985. 144 с. [Annin B. D., Bytev V. O., Senashov S.I. Group properties of elasticity and plasticity equations. Novosibirsk: Nauka. Sibir. Otd., 1985. 144 pp.]

3. Андреев В.К., Бублик В.В., Бътев В. О. Симметрии неклассических моделей гидродинамики. Новосибирск: Наука, 2003. 351 с. [Andreev V.K., Bublik V. V.; Bytev V.O. Symmetries of nonclassical models of hydrodynamics. Novosibirsk: Nauka, 2003. 351 pp.] 
4. Савин Г. Н. Распределение напряжений около отверстий. Киев: Наукова думка, 1968. 888 c. [Savin G. N. Stress Distribution around the holes. Kiev: Naukova Dumka, 1968. 888 pp.]

Поступила в редакцию 21/XII/2011;

в окончательном варианте - 12/II/2011.

MSC: 74B20

INVESTIGATION OF THE STRESS-STRAIN STATE OF THE ASYMMETRICAL ELASTIC PLATES

\section{V.O.Bytev, I. V. Slezko}

Tyumen State University,

10, Semakova st., Tyumen, 625003, Russia.

E-mail: slezkoirina@rambler.ru

The two-dimension model of the deformation of asymmetrical elastic solid is considered in the article. The exact solution of flat asymmetrical elasticity's problem about simple shear in the plate which is weakening by hole is demonstrated. Comparative analysis of the obtained solution with the classical ones are given.

Key words: asymmetrical elasticity, deformation of the plate, the simple shear.

Original article submitted 21/XII/2011; revision submitted $12 / \mathrm{II} / 2011$.

Vladislav O. Bytev (Dr. Sci. (Phys. \& Math.)), Head of Dept., Dept. of Mathematical Modelling. Irina V. Slezko (Ph. D. (Phys. \& Math.)), Associate Professor, Dept. of Mathematical Modelling. 